\title{
Managing Uncertainty in Chronic IIIness From Patient Perspectives
}

\author{
Amanda Brown, $\mathrm{PhD}$ \\ Walden University, Minneapolis, Minnesota, United States \\ iD https://orcid.org/0000-0003-2359-0923 \\ Susan Hayden, PhD \\ Walden University, Minneapolis, Minnesota, United States
}

Karen Klingman, $\mathrm{PhD}$

Upstate University Hospital, Syracuse, New York, United States

Leslie C. Hussey, PhD

Walden University, Minneapolis, Minnesota, United States

Contact: brownama@upstate.edu

\begin{abstract}
Chronic illness is an increasing concern in the United States as the Centers for Disease Control and Prevention reports that $50 \%$ of adults have at least one chronic illness. When individuals must adjust to chronic illness, they may experience uncertainty regarding the illness, prognosis, and symptoms. The purpose of this qualitative, phenomenological study was to explore the management strategies of uncertainty in chronic illness from patients' perspectives. Merle Mishel's uncertainty in illness theory was the framework used to explore the lives of individuals diagnosed and living with chronic illness. In-depth interviews were conducted with eight individuals who have chronic disease. Results revealed themes that individuals with chronic illness do experience uncertainty and that it impacts almost all aspects of their lives. Some of the themes identified were chronic illness, uncertainty, impact of uncertainty on daily living, coping or managing uncertainty, and communication from the healthcare team about uncertainty. The individuals expressed changes in daily activities and retraining their minds to accept their new normal. This study offers coping and management strategies that healthcare professionals can implement into treatment plans to improve the quality of life for individuals with uncertainty in chronic illness. The information produced from this study will aid healthcare providers in understanding the need to address the uncertainty at diagnoses and offer ways to assist patients in coping and managing uncertainty.
\end{abstract}

Keywords: chronic illness; patients' experience; lived experience; coping

Date Submitted: June 3, 2019 | Date Published: March 17, 2020

\section{Recommended Citation}

Brown, A., Hayden, S., Klingman, K., \& Hussey, L. C. (2020). Managing uncertainty in chronic illness from patient perspectives. Journal of Excellence in Nursing and Healthcare Practice, 2, 1-16. https://doi.org/10.5590/JENHP.2020.2.1.01 
Brown et al., 2020

\section{Introduction}

The incidence of chronic illness is a serious healthcare concern in the United States. The Centers for Disease Control and Prevention (CDC) reports that half of U.S. adults have at least one chronic illness (as cited in Fleming et al., 2015). Chronic illness, also known as chronic disease, is used to describe illnesses that can be treated but have no cure. Individuals with chronic illnesses can experience uncertainty and the inability to place meaning to illness-related events, causing significant psychological stress (Sajjadi et al., 2015). Uncertainty in illness is defined as a cognitive state characterized by the individual's inability to establish the meaning of events related to the illness (Giammanco et al., 2015). Individuals living with chronic illnesses may experience uncertainty when the cause of the disease or disease progression is unknown, when symptoms fluctuate and are unpredictable, and when there is a lack of knowledge about treatment options and outcomes. High levels of uncertainty can lead to higher levels of perceived stress, depressive symptoms, as well as poorer emotional well-being, which, in turn, can lead to inability to function, difficulty maintaining relationships, confusion, misunderstanding, and decrease quality of life (Kurita et al., 2013). Unawareness of uncertainty and how to manage it can lead to overuse of healthcare facilities, inappropriate health-seeking behaviors, and overall poor outcomes for the individuals (Bennell \& Taylor, 2013). The confusion and misunderstanding that result from uncertainty can detract from the timely and necessary illness management (Sav et al., 2015). In chronic illness, uncertainty and its management are crucial aspects that influence the individual's quality of life (Bloom et al., 2008; Germino et al., 2013; Kornblith et al., 2007). Individuals living with chronic illness do not always know how, when, or if symptoms will appear (Mishel, 2014). The purpose of this study was to explore the stories of the lived experiences of those living with chronic illness and analyze the approaches used to cope or to manage uncertainty. This will assist in the development of informed recommendations and consistent methods used by nurses and medical professionals to include in individuals' treatment plans upon diagnosis. Providing patients with strategies to manage the uncertainty could potentially improve the patients' quality of life.

\section{Background}

The $\mathrm{CDC}$ reports that $50 \%$ of all adults are diagnosed with at least one chronic illness and one in four have two or more chronic illnesses (CDC, 2014; Center for Managing Chronic Illness, 2011; Fleming et al., 2015). Care for individuals with chronic illnesses is currently a top concern raised by the national health agendas and efforts have been put forth to increase self-management and to promote self-care (World Health Organization, 2009, as cited in Martinez-Marcos \& De la Cuesta-Benjumea, 2015). Chronic illness not only affects the patient but the family, caregivers, and friends who surround the individual, including the public. In 2015, caring for chronic illnesses consumed an estimated $75 \%$ of the U.S. health care costs (Fleming et al., 2015).

Once diagnosed, individuals often undergo surgery, specific treatments, and pharmacological therapies. Following the interventions, the course of treatment and disease process can remain uncertain (Giammanco et al., 2015). The way individuals cope with the uncertainty of the disease process, as well as symptoms and diagnosis can vary (Coderey, 2015). When a coping strategy is found to produce a positive experience, individuals' health and quality of life have been found to improve (Kim et al., 2016).

Little research has been conducted on interventions used to cope with uncertainty. Hoth et al. (2013) suggested further research is necessary to examine different factors that contribute to positive, effective coping strategies. For example, factors such as social environment can impact the management of uncertainty and early intervention at diagnosis can improve patient outcomes and quality of life. Medical and nursing professionals can identify the uncertainty, but patients must have effective coping methods to maximize efficacy of medical treatments. According to Borneman et al. (2014), a case report demonstrated that a woman 
struggled with her diagnosis and uncertainty and explained it as being similar to being stuck in a "black hole" of questions but being offered no acceptable answers. It is important for healthcare professionals to be aware of uncertainty and the impact it has on patients. Not properly managing uncertainty can lead to further adverse consequences. Patients with chronic illnesses constantly experience uncertainty, which can damage physical, social, spiritual, cognitive, psychological, and economic aspects of life and daily activities (Sajjadi et al., 2015).

\section{Theoretical Foundation}

Merle Mishel's uncertainty in illness theory (UIT) has informed this work. The UIT advocates that uncertainty occurs when a person is unable to categorize the meaning of illness-related events, such as having difficulty interpreting their physical symptoms or predicting the likely outcome of treatment (as cited by Hoth et al., 2013, p. 1171). Uncertainty is a common cognitive state amongst those with chronic illness and the UIT addresses uncertainty related to the diagnostic and treatment phases of an illness with poor expected outcomes (Mishel, 1988, 2014). Later, Mishel developed the reconceptualized uncertainty in illness theory (RUIT) to address the constant uncertainty associated with chronic illness requiring long-term management or illnesses with the possibility of recurrence (Mishel, 1990, 2014). The preferred outcomes from the RUIT are growth and adapting to a new value system. This is opposed to the UIT, which centers on returning to a previous level of functioning (Mishel, 1990, 2014).

Three factors can lead to uncertainty, the theoretical concept. The first is biological factors, including illness severity, symptom pattern, and symptom familiarity. The second is psychological factors, such as learned helplessness, emotional state, and cognitive distortions. The third is social factors, which can include support systems and education level. Consequences of uncertainty are anxiety, depression, fear, agitation, and anger. All of the above consequences can lead to maladaptive coping, increased psychological stress, and information seeking. The UIT provides a framework that focuses on the selection of interventions geared toward the psychological and behavioral outcomes of individuals struggling with the conditions of uncertainty (Germino et al., 2014).

\section{Literature Review}

Chronic illness is continuing to increase worldwide along with the demand to improve the care of those diagnosed with a chronic illness (Gibbons et al., 2017). Examples of chronic illness include but are not limited to arthritis, diabetes, cancer, and coronary heart disease. Treating and caring for individuals with chronic illnesses has become a healthcare priority as the increasing prevalence adds stress to not only the patient but also the healthcare system (Gibbons et al., 2017). Researchers have suggested that individuals with chronic illness face uncertainty including significant disruption to family life, well-being, and quality of life as they experience unpredictable and incurable conditions (Hurt et al., 2017). Day-to-day living requires endless decisions which require some degree of certainty for humans to continue to move forward (Brizi, Mannetti, \& Kruglanski, 2016). Not knowing if and to what extent symptoms may appear can leave individuals secluded in their homes in fear of extreme exacerbations. Uncertainty can result in a great loss of change in the individual's ability to take pleasure in daily activities (Suzuki, 2012), which can often lead to further problems such as depression (Fuemmeler et al., 2001; Gentes \& Ruscio, 2011; Jiang \& He, 2012).

There is a lack of literature on how those with chronic illness manage uncertainty. Healthcare providers are expected to mediate the impact of uncertainty while also assisting in limiting and preventing the negative consequences (Mishel, 2010; Nanton et al., 2016). 
When using a phenomenological approach, in nursing research, the desire is to gain a deeper understanding of the patients' perspectives on illness, suffering, and the care provided (Derico, 2017). Bennell and Taylor (2013) used a descriptive phenomenological approach to examine the experiences of individuals living with gastroparesis, a chronic illness. The authors interviewed nine individuals living with gastroparesis; four main themes resulted from the interviews, and it was concluded from the in-depth interviews that the chronic illness affects all aspects of the individuals' lives and the uncertainty that the individual's experience can trigger psychological distress and feelings of loss (Bennell \& Taylor, 2013). Researchers Inan et al. (2016), used a phenomenological approach to describe the experiences of women in Turkey as they were going through the diagnostic phase of breast cancer. The data for the study were collected through semistructured, in-depth interviews of nine subjects. Four themes were identified from the interviews: facing the diagnosis, uncertainty, avoidance, and holding on to life (Inan et al., 2016). The authors were also able to conclude that breast cancer patients experience uncertainty about maintaining their role at home as part of the Turkish culture as well as how cancer is viewed within the culture. The methodology of the study allowed the authors to explore into the lives of the women and better understand the lived experiences and how health care professionals should be aware of how the patients psychologically adapt and how to guide them with new information and support.

Using a phenomenological approach with purposive sampling, Coventry et al. (2014) explored bodily and emotional consequences of physical and mental multimorbidity in lived time and lived space amongst individuals with different combinations of mental-physical multimorbidity. The researchers recruited subjects with chronic obstructive pulmonary disease from three acute hospitals in Greater Manchester, United Kingdom. It was concluded that the lifeworld of those with multimorbidity was characterized by temporal and spatial contraction, meaning that individuals exhausted their energy on coping with symptoms and did not have energy left to spend on social aspects of life (Coventry et al., 2014).

The examples provide insight into the usefulness of the phenomenological method and benefits of such indepth interviews toward understanding the lived experiences of those with chronic illness. The trend observed while searching the literature is the majority of studies use the Mishel Uncertainty in Illness Scale (MUIS) to determine the level of uncertainty experienced by the individuals. Mishel (1981) confirmed the MUIS as a useful instrument after undergoing a double-factoring procedure with both resulting in high reliability. Validity of the scale was confirmed by construct and convergent validity (Mishel, 1981).

For instance, Jian and He (2012) used the MUIS to determine the effects of an uncertainty management intervention for individuals diagnosed with chronic obstructive pulmonary disease on uncertainty, anxiety, depression, and quality of life. The use of the MUIS showed that when results were compared the intervention group showed a greater improvement in uncertainty than the control group. Jeon et al. (2016) studied the relationship between perceived gastrointestinal symptoms, uncertainty, and recovery in individuals diagnosed with gastric cancer postgastrectomy. Through the use of MUIS, the researchers were able to assess uncertainty levels in comparison to perceived recovery time and determined that health care providers should promote recovery and take into account patients' socioeconomic factors, income, and education when providing information and counseling to patients to reduce the level of uncertainty (Jeon et al., 2016).

Another study used MUIS to capture how participants viewed the cues about their cancer. The data discovered that interventions to address avoidance and the intolerance of uncertainty in those diagnosed with lung cancer might help improve the psychological adjustments experienced (Kurita et al., 2013). An inherent weakness that has been observed in the literature is the lack of studies exploring the uncertainty and how it affects the subjects' quality of life and how the individuals cope and manage that uncertainty. The literature has supported that individuals with chronic illness do experience uncertainty and that health care providers should be addressing the uncertainty at the time of diagnosis. The literature also supports the notion that 
when educating the individuals, it is important to take into account the work, financial, and educational status of the patients (Suzuki, 2012) and that intervention can reduce the levels of uncertainty. Studying how the uncertainty impacts individuals over a length of time and how to manage it remains weak in the literature. Perhaps this is because the UIT, supported by quantitative studies and through the use of MUIS, has been given a great deal of attention over the RUIT, which is supported by qualitative studies.

A study by Germino et al. (2013) was located that developed an uncertainty management intervention that was developed and tested in clinical trials for breast cancer patients. Germino et al. (2013) recruited two groups of subjects, one intervention group and one control group of breast cancer patients. The intervention group was provided behavioral strategies to control uncertainty and to promote self-efficacy while coping with recurrence, calming self-talk, breathing, relaxation, and distraction to deal with triggers of uncertainty. A second session was developed based on the current literature of young breast cancer survivors with content that focused on hormone changes, disturbances in body and self-image, fatigue, and prelymphedema symptoms (Germino et al., 2013). The researchers found that those in the intervention group reported lower levels of uncertainty because the subjects felt educated and supported. In chronic illness how one copes and manages uncertainty can greatly impact the individuals' quality of life and outcomes. Exploring the lived experiences of those living with uncertainty in chronic illness is meaningful because the literature lacks in providing meaning into the lives of those living with chronic illness and how the individuals cope. There are strategies for decreasing the uncertainty, education, medication, and treatment side effects, but understanding the methods used by the individuals is the goal of the study.

Lastly, Giammanco et al. (2015) conducted a study to validate the MUIS as a method to assess and quantify uncertainty in individuals with chronic illness. The researchers concluded that the tool is useful and suggested the need for further study into the strategies used for coping with uncertainty as patients search for clarity of treatment. Through the use of the MUIS, researchers are able to measure the level and intensity of the uncertainty but little is known about the experiences of uncertainty. The literature does not provide evidence as to how individuals cope with uncertainty or manage the uncertainty of not knowing how the illness will impact daily activities. The purpose of this study was to explore the management of uncertainty in chronic illness and strategies used from patient perspectives.

\section{Method}

\section{Design}

A qualitative methodology, transcendental phenomenology, was selected for this study to understand the uncertainty experienced by individuals diagnosed with chronic illnesses. For this study chronic illness, also known as chronic disease, was defined as a condition that can be treated but not cured (Center for Managing Chronic Illness, 2011; Fleming et al., 2015). Due to limited research on this topic, the focus included uncertainty in any chronic illnesses rather than selecting a specific illness. This allowed for a larger population pool to obtain an adequate number of participants from the study. Data were collected from individuals diagnosed with a chronic illness until no new trends or patterns were expressed, and saturation was met.

\section{Assumptions}

One assumption was that the participants will provide honest, real answers during the interviews and best describe their lived experience with chronic illness to the best of their ability. To reduce the concern for bias, results were reviewed by two other reviewers. The second assumption of this study was that uncertainty in chronic illness is not a desirable state and participants desire to manage uncertainty in chronic illness. 


\section{Sample}

A recruitment poster was publically posted on Facebook and Instagram and in the waiting room of a family practice. The poster for recruitment, included inclusion criteria, benefits of the study, and contact information. Inclusion criteria were being at least 18 years old, English speaking, and diagnosed with at least one chronic illness for longer than 6 months. Exclusion criteria were a recent diagnosis (i.e., acute illness rather than chronic) and inability to verbalize understanding and experiences of uncertainty. Interested participants contacted the researcher, who then screened participants. If the participant met the criteria a consent was emailed to the participant with instructions to review, sign, and send back. After a willing participant signed the consent, and emailed it back, a participant number was assigned and the interview was set up. Prior to the start of the interview uncertainty was discussed and defined along with a verbal discussion reviewing consent. Following the consent discussion inclusion questions were asked using the interview guide created to help structure the interviews. The interview guide consisted of demographic questions and open ended questions to help facilitate discussion about uncertainty and how it impacts daily activities. If at that point the participant expressed no experience with uncertainty or did not meet inclusion criteria, the interview was concluded.

A combination of criterion and purposive sampling strategies were utilized to obtain a variety of experiences associated with a range of chronic illnesses. The goal was to recruit participants with different gender and ages along with different chronic illnesses.

Of the 14 people who expressed interest in participating in the study, 11 sent an email for communication and retrieval of the consent. Eight of those volunteers scheduled an interview, met the inclusion criteria, and ultimately participated in the study. There were no personal or professional relationships between the researcher and participants that would impact the outcomes.

\section{Data Collection}

The interview options were face to face, FaceTime, or telephone. Three interviews were via FaceTime, one started out on FaceTime but because of poor connection, the interview concluded via telephone. Two participants stated they preferred to be interviewed via telephone, and the remaining three did not have FaceTime capabilities and were interviewed via telephone. The interviews lasted between 15 and 40 min and were conducted by one researcher. Prior to the interviews, each participant was provided a definition of uncertainty and chronic illness and each provided verbal assurance that they understood. The participants were asked to provide their own definition of uncertainty and provide one example of an experience. An interview guide was used to start the conversations and ensure all participants were asked the same questions as they fit into the discussion. Some of the questions from the interview guide were, "How does uncertainty impact your daily routine and activities of daily living?" "How much of your time is spent thinking about uncertainty or how much time would you say is spent living in an uncertain state?" and "What are some daily rituals or habits, if any, that you find help manage your uncertainty, or help improve the chances that you will have a good day?"

\section{Ethical Considerations}

Permission for human subject research was established through the Walden University Institutional Review Board prior to recruitment efforts. All participants were 18 years or older and English speaking. Ethical concerns consisted of ensuring their health status was such that he or she was able to consent. Consent was sent via secure email server and participants were asked to sign, scan, and email back to researcher. Consent was reviewed prior to the start of the interviews and all participants agreed to be audio recorded. Data 
collected remained confidential, participants were given a participant number and names were not documented or recorded. No personal identifiers were documented and all data was stored on a password protected computer.

\section{Data Analysis}

All data obtained from the interviews were stored, coded and transcribed using NVivo Version 11, computer assisted qualitative data analysis software (Houghton et al., 2017), by one researcher. Once the data were stored and organized in NVivo, "fracturing" was used to break down the data and organize them into categories, to develop themes or categories across all cases (Polit \& Beck, 2012, p. 565). Themes and subthemes, nodes, could then be extracted (Houghton, et al., 2017). The data were then coded, key terms and patterns identified, trends, themes, and categories were discovered. Results were reviewed by two committee members.

Nodes were categorized based on questions developed for the interview guide and common themes brought up by the participants during the interviews to best explore the lived experiences of individuals with chronic illness. The nodes offered a strategy to break down the interviews for interpretation.

\section{Rigor}

Participants with different chronic illnesses were interviewed offering a variety of experiences with symptoms, treatments, and outcomes. Member checks included restating what the participant stated to confirm understanding if it was not felt it was understood correctly (Polit \& Beck, 2012). Interviews were also recorded and transcribed verbatim. All participants received results to review and approve.

\section{Findings}

All participants had experience with uncertainty and had been undergoing treatment for one or more chronic illnesses for over a year. Chronic illnesses represented in the study sample included atrial fibrillation, arterial sclerosis, interstitial cystitis, asthma, gastroparesis, Ehlers-Danlos syndrome, sphincter of oddi, celiac disease, pancreatic insufficiency, and new daily persistent headache (diagnosed over 6 months prior). Participants were all female, ranged from 20 to 70 years old, spoke English, and were from the United States (see Table 1).

The interview questions guided the discussions from which six main themes emerged. The participants described their chronic illness or illnesses including length of time diagnosed, experience with uncertainty, and the impact of uncertainty on the daily lives, coping or managing strategies for uncertainty, communication from healthcare teams about uncertainty, and important information about uncertainty and chronic illness from their own experiences. The developing nodes (see Appendix) grouped all comments as related to themes. 
Brown et al., 2020

Table 1. Characteristics of Participants

\begin{tabular}{lc}
\hline \multicolumn{1}{c}{ Characteristic } & Sample $(\boldsymbol{n}=\mathbf{8})$ \\
\hline Chronic illness type & 1 \\
Asthma & 1 \\
Atrial fibrillation & 5 \\
Gastroparesis & 1 \\
Sphincter of Oddi & 1 \\
Arteriosclerosis & 1 \\
Interstitial cystitis & 1 \\
Celiac disease & 1 \\
Pancreatic insufficiency & 1 \\
New daily persistent headache & 1 \\
Ehlers-Danlos syndrome & \\
Age & 3 \\
$21-30$ & 2 \\
$31-40$ & 1 \\
$41-50$ & 1 \\
$51-60$ & 1 \\
$61-70$ & \\
\hline
\end{tabular}

\section{Node 1: Chronic Illness}

Participants shared chronic illnesses with which they were diagnosed, the length of time it took to be diagnosed, and how long they have been diagnosed. For example, Participant 1 said, "Atrial fibrillation and arterial sclerosis, symptoms started two year ago." Participant 2 stated, "I have been diagnosed with asthma about ten years ago: it took about five years to come to a diagnosis.” According to participant 3 ,

Interstitial cystitis, symptoms started when I was 12 years old, for the longest time no one knew what was going on, I got sick of it and did research on my own, the first doctor did not listen and I finally found an NP that was open to listening and agreed that I had interstitial cystitis.

The first three participants struggled to get a diagnosis. Participants 1 and 3 shared that they had to "bring possible diagnoses to the doctors and do the research themselves." Participant 2 continued to listen to the doctors and did not push for further testing until an advocate stepped in and demanded further steps be taken.

Participant 4 stated, "I have had new daily persistent headache since 2009 and I was diagnosed with gastroparesis, symptoms started at the age of 16 and was just recently diagnosed at 22." This is a unique case in that the individual has a psychiatric history and shared the delay in diagnosis was because the doctors brushed symptoms off as psychological and not physical.

Participant 5 said, "I have been diagnosed with gastroparesis Ehlers-Danlos syndrome.” Participant 6 explained, "I am currently being treated for gastroparesis but I have never had any tests done, my doctor is just treating me off observation and seeing what works." Participant 7 received a quick diagnosis: "I was diagnosed with gastroparesis in two months in 2013." She also shared that she had "brought the tests she 
wanted to have done to the doctors and insisted on having them and that is how a diagnosis was determined so rapidly."

According to Participant 8, "I have been diagnosed with sphincter of Oddi dysfunction, severe gastroparesis, pancreatic insufficiency and celiac disease in 2012, with symptoms starting in 2008 after a gallbladder surgery." She shared the "long journey from the start of symptoms to almost losing her life." She spent almost a year between the hospital and nursing home, had to travel to seek the best care and had to uproot her youngest child to get what was needed.

After listening to the participants' diagnoses and the process each went through to get them, it is clear that either having an advocate with you or being your own advocate is critical in obtaining a diagnosis.

\section{Node 2: Uncertainty}

Participants were asked to share their experiences with uncertainty. Participant 1 said,

I met with my cardiologist after having symptoms and my right leg gave out, things progressed slowly. I started treatment with my primary care doctor and was put in physical therapy, this did not work. I was very weak and very tired. With each treatment I thought I was going to be cured, the doctors still do not know what was happening to me. I fear that I may not ever be able to get out of the house.

Participant 3 explained, "for the longest time, no one was certain about what was going on, so I had to figure it out on my own, I remember dealing with a urologist and she did not believe me that I have interstitial cystitis and so she was kind of like insulting me." Participant 5 stated that "I experience a lot of uncertainty about what my quality of life will be like in the future."

Fear of the unknown was a common statement, fearing if symptoms would worsen, fear of leaving the house, and fear of never going back to the life lived prior to chronic illness. All participants shared feelings of uncertainty during the diagnosis process and after being diagnosed.

\section{Node 3: Impact of Uncertainty on Daily Activities}

The impact that the uncertainty has on each of the participants provided insight into the fact that symptoms, fear, and the unknown can prevent individuals with chronic illness and uncertainty from even leaving the house or partaking in daily activities.

Participant 1 stated that

when not in A fib I have a very normal day but I never know if I am going to wake up and not be able to walk or get up and down the stairs, I am asleep by $3 \mathrm{pm}$, I miss out on family events because I am just too exhausted and I do not know if it will just come on, so then I have to cancel. Just walking becomes difficult so I do not want to venture off too far alone in case I just go into A fib.

Participant 3 shared that she "just dealt with the pain; nobody knew what was causing it or how to control it." Participants also shared the need to wake up earlier to take medications, sit up ensuring the stomach is settled, and to schedule in rest time because it is too exhausting to put make up on and get dressed. Participant 6 said, "My entire day is built around uncertainty and my symptoms because at any point I can become very very nauseous or I can become very very sick.” 
Participants also shard the inability to keep plans with friends and family. According to Participant 7, "I mostly stay at home, I can never say $100 \%$ I will be there, I am just too nervous about the consequences if I push the limit even just a little bit.” Participant 4 said, "I cannot make plans, I am always bailing.”

There are also psychological impacts from the uncertainty; participants shared increased levels of anxiety and depression. "I spent so much time in tears of frustration because there were no concrete answers for what was going on" (Participant 8).

Node 3 provided insight into the physical and psychological factors that results from uncertainty and chronic illness. Factors include the planning of daily activities, fear of leaving the house, how the chronic illness affects the family, and the inability to work or hold a job.

\section{Node 4: Coping or Managing Uncertainty}

After the participants explained how they were impacted by uncertainty and chronic illness, they each provided detail about their own coping and management strategies. The most common strategies included waking up early in the morning and planning ahead. Several of the participants shared that they have to make time to take medications, including time to sit up to allow everything ingested to digest to prevent symptoms. One participant described the need to wake up early to get ready, as frequent breaks are required during the process because of fatigue.

Other coping strategies included advocating for themselves and doing their own research and presenting it to the physicians. Some shared "frustration in needing to do this information seeking, while some felt doctors just do not have time to do it." Participant 6 felt having to do this research on her own was "not her responsibility and that is what the doctors were for. She then lost confidence in her doctor because she had to take these steps. "It is just a lot of googling and taking care of myself."

Two participants shared that after diagnosis a team of doctors came together and they were started in therapy to address the psychological aspect of uncertainty and chronic illness. "It has been instrumental, I honestly do not know if I would be here today if it was not for being in therapy and finding a good therapist that has experience with chronic illness" (Participant 7). Interestingly, the two who participated in therapy shared that "retraining their brains" was key to being able to cope and manage uncertainty. "I had to retrain my brain to be accepting of the fact that I do not know what tomorrow will be like and do not know what later today will be like" (Participant 7). "Every day I have to embrace it as my new normal so I get up and do what I have to" (Participant 8).

One participant shared that she rides horses and another tried to exercise every day to build muscle to help with recovery. Other participants discussed the need to avoid certain workouts and utilize the trial and error method to determine what works for them to cope and manage uncertainty.

\section{Node 5: Communication With Healthcare Team About Uncertainty}

The majority of participants were quick to answer the question whether the health care team discussed uncertainty with them or not. Most responded with "no." Participant 6 stated, "no, absolutely not, never, I mean in a sense of their own uncertainty about what is wrong with me or what is causing it but never in relation to me feeling uncertain." Several shared that the doctors would often give a flyer with information about the illness on it, but not go over any management strategies or give a medication, and ask the patient to return for a follow-up to see if symptoms improved. Participant 5 stated, 
The doctors they just give me a quick summary like yeah, we saw this on your last test and we are giving you this drug and I do not understand what it is. They do not explain what the test results came back showing and why they want to do what it is they are suggesting, like not understanding the rationale behind my care creates greater anxiety.

Those who were in therapy did have medical teams who discussed uncertainty with them and that is how the psychological aspects were addressed-doctors worked with the participants as a team approach to care. Participant 8 stated,

Yes, there was the talk of uncertainty with the doctors; they were very clinical about it; when I was at the med center they set me up with a psychiatrist and a psychologist. Mostly because my stomach was not functioning and my brain was not getting proper messages that I needed so they treated me for the depression and my dosage has since been going down as I am getting more nutrition.

\section{Node 6: Important Information From Participants}

At the conclusion of all interviews, each participant was asked if there was anything they would like to add or that they felt would be helpful for the study. A common response was communication within the healthcare team and to not treat every case like the other, be understanding that with chronic illness not all cases present the same.

Other responses were for health care providers to explain using common terms and not medical jargon. "The patient will be able to understand what it is that is happening to them and what the proposed plan is" (Participant 5). Participants also shared it is important for individuals with chronic illnesses to learn that they do not have control over the sickness and that it is important for individuals with chronic illnesses to learn that they do not have control over the sickness and that it is important to maintain a positive attitude.

\section{Discussion}

All participants in this study expressed that they were impacted by uncertainty in some form either psychologically or physically. From the start of symptoms, participants expressed feelings of frustration with being dependent on the doctor to know what was wrong and how to fix it. Many experienced a trial and error process of medications in hopes one would work and fix the symptoms. In some cases, symptoms were controlled within a finite timeframe, while in other cases, the struggle to find an effective treatment continued over time.

Even when symptoms were controlled, fear of the treatment no longer working and fear of the illness worsening remained. Participants discussed how uncertainty was present every day in relation to searching for a diagnosis, enduring and anticipating outcomes of diagnostic and treatment procedures, and what the future would hold.

Although uncertainty negatively impacted quality of life, participants developed effective coping strategies such as finding a routine, researching symptoms on their own, exercising, and planning ahead. Some participants found spiritual rituals and psychotherapy helped manage their uncertainty. Participants who were treated by an interdisciplinary team of medical and mental health providers were able to adapt to their uncertainty by retraining their brains to create a new normal. This was achieved by reading and/or working with psychologists on coping methods. 


\section{Implications for Future}

Patient-provider communication and trust were also found to be important factors in coping and managing the uncertainty associated with chronic illness. For example, participants suggested that if doctors would use common language and clearly explain treatment plans and diagnoses patients would feel less uncertainty and be less inclined to seek out information on their own. Participants suggested that if the healthcare team would respect self-researched information brought to them by patients, a better level of patient-provider trust would help alleviate the distress of uncertainty. Participants suggested providers improve the explanation of treatment plans, get to know each patient personally, and treat each patient individually based on their needs. To cope with patient-provider communication issues, participants felt a need to be accompanied by an advocate at appointments to help reinforce the need for ongoing care.

The findings from this study support and enhance the current body of knowledge regarding uncertainty and chronic illness. For example, Caruso et al. (2014) found that individuals with chronic illness experience uncertainty may not understand the meaning of events linked to the illness and are unable to predict when events will occur (Caruso et al., 2014). Bennell and Taylor (2013) concluded that chronic illness affects all aspects of life and the associated uncertainty can trigger psychological distress. This study supports the findings of Caruso et al. (2014) and Bennell and Taylor (2013) and further reveals that individuals with chronic illness experience psychological distress associated with uncertainty about their health, but also that the uncertainty of chronic illness impacts family systems, ability to work, and planning daily activities. For participants in this study, uncertainty often caused fear which in turn led to self-seclusion and inability to work.

The establishment of a new normal coping mechanism for uncertainty, as identified in this study, supports Mishel's (2014) RUIT, which describes individuals with chronic or recurring illness requiring frequent treatment. Mishel (2014) evolved the RUIT from the previous UIT to account for changes associated with chronic illness and the need to adapt to a new norm. According to the RUIT, when individuals continue to feel uncertain they lose tolerance and disorganization begins to take over. As the perspective on disorganization changes, individuals with chronic illness evolve their perspective from what they used to have predictability and control, to a new life with changes and unforeseen events (Mishel, 2014), that is, a new normal.

\section{Limitations}

There were limitations to this study. First, all participants were female, offering no variation in gender. Men were not purposely excluded but none offered to participate. Another limitation was the inability to use FaceTime for all calls. Obtaining observational data on all participants would have added to the rigor of the study.

\section{Relevance to Clinical Practice}

The results of the study are supported by the literature that individuals with chronic illness do experience uncertainty. Through in-depth interviews, a better understanding of the lived experiences of uncertainty and what individuals with chronic illness experience from the beginning of symptoms to the management of their new normal. Self-care knowledge is important in managing uncertainty in chronic illness as confirmed by Hoth et al. (2013), who stated that self-care knowledge is a strategy to improve patient care and outcomes. The study has implications for social change by providing medical professionals a glimpse of what patients with chronic illness experience while trying to manage uncertainty. A unique contribution of the study was the concept and validation of the multidisciplinary team approach. Using an interprofessional collaboration of specialist can improve the uncertainty for individuals with chronic illness. Implications for future research 
include focusing on gender variation, observation during interviews, delving into the process for creating a specialist team, and timeline for treatment interventions.

\section{Conclusions}

Chronic illness is an increasing concern in the United States (Fleming et al., 2015). With increasing individuals seeking treatment for chronic illnesses, strategies for care need to be altered to fit the needs of the chronically ill. There will not always be a test for a diagnosis or definitive answer. This study provided the detailed experiences of eight individuals with chronic illness and uncertainty. The participants hoped to share their stories to improve future patient care and outcomes. As more healthcare providers become aware of how individuals with chronic illness perceive their care, they may adopt new strategies to improve the process.

\section{References}

Bennell, J., \& Taylor, C. (2013). A loss of social eating: The experiences of individuals living with gastroparesis. Journal of Clinical Nursing, 22(19-20), 2812-2821. https://doi.org/10.1111/jocn.12196

Bloom, J. R., Stewart, S. L., D’Onofrio, C. N., Luce, J., \& Banks, P. J. (2008). Addressing the needs of young breast cacner survivors at the five-year milestone: Can a short-term, low-intensity intervention produce change? Journal of Cancer Survivorship, 2, 190-204. https://doi.org/10.1007/s11764-008$\underline{0058-x}$

Borneman, T., Irish, T., Sidhu, R., Koczywas, M., \& Cristea, M. (2014). Death awareness, feelings of uncertainty, and hope in advanced lung cancer patients: Can they coexist? International Journal of Palliative Nursing, 20(6), 271-277. https://doi.org/10.12968/ijpn.2014.20.6.271

Brizi, A., Mannetti, L., \& Kruglanski, A. W. (2016). The closing of open minds: Need for closure moderates the impact of uncertainty salience on outgroup discrimination. The British Journal of Social Psyschology, 55(2), 244-262. https://doi.org/10.1111/bjso.12131

Caruso, V., Giammanco, M. D., \& Gitto, L. (2014). Quality of life and uncertainty in illness for chronic patients. Mediterranean Journal of Clinical Psychology, 2, 2. https://doi.org/10.6092/2282$1619 / 2014.2 .990$

Centers for Disease Control and Prevention (CDC). (2014). Chronic disease and health promotion. https://www.cdc.gov/chronicdisease/overiew/index.htm

Center for Managing Chronic Illness. (2011). What is chronic disease? University of Michigan. http://cmcd.sph.umich.edu/what-is-chronic-disease.html

Coderey, C. (2015). Coping with health-related uncertainties and risk in Rakhine (Myanmar). Health, Risk \& Society, 17(3/4), 263-284. https://doi.org/10.1080/13698575.2015.1096327

Coventry, P. A., Dickens, C., \& Todd, C. (2014). How does mental-physical multimorbidity express itself in lived time and space? A phenomenological analysis of encounters with depression and chronic physical illness. Social Science \& Medicine, 11, 8108-8118.

https://doi.org/10.1016/j.socscimed.2014.07.068

Derico, S. P. (2017). The use of phenomenology in nursing education: An integrative review. Nursing Education Perspectives, 38(6), E7-E11. https://doi.org/10.1097/01.NEP.0000000000000216 
Fleming, A. R., Phillips, B. N., \& Kline, K. M. (2015). Clients with chronic illness in VR agencies: How do they compare with other client population? Journal of vocational Rehabilitation, 42(2), 101-113. https://doi.org/10.3233/JVR-150728

Fuemmeler, B. F., Mullins, L. L., \& Marx, B. P. (2001). Posttraumatic stress and distress among parents of children surviving a brain tumor. Child Health Care, 30, 169-182. https://doi.org/10.1207/S15326888CHC3003 1

Gentes, E. L., \& Ruscio, A. M. (2011). A meta-analysis of the relation of intolerance of uncertainty to symptoms of generalized anxiety disorder, major depressive disorder, and obsessive-compulsive disorder. Clinical Psychology Review, 31, 923-933. https://doi.org/10.1016/j.cpr.2011.05.001

Germino, B. B., Mishel, M.H., Crandell, J., Porter, L., Blyler, D., Jenerette, C., \& Gill, K. M. (2013). Outcomes of an uncertainty management intervention in younger African American and Caucasian breast cancer survivors. Oncology Nursing Forum, 4O(1), 82-92. https://doi.org/10.1188/13.ONF.82-92/

Giammanco, M. D., Gitto, L., Barberis, N., \& Santoro, D. (2015). Adaptation of the Mishel Uncertainty of Illness Scale (MUIS) for chronic patients in Italy. Journal of Evaluation in Clinical Practice, 21(4), 649-655. https://doi.org/10.1111/jep.12359

Gibbons, C. J., Small, N., Rick, J., Hann, M., \& Bower, P. (2017). The patient assessment of chronic illness care produces measurements along a single dimension: Results from a Mokken analysis. Health and Quality Life Outcomes, 15(1), 61. https://doi.org/10.1186/s12955-017-0638-4

Hoth, K. F., Wambodlt, F. S., Strand, M., Ford, W. D., Sandhous, R. A., Strange, C., Bekelman, D. B., \& Holm, K. E. (2013). Prospective impact of illness uncertainty on outcomes in chronic lung disease. American Psychological Association, 23(11), 1170-1174. https://doi.org/10.1037/a0032721

Houghton, C., Murphy, K., Meehan, B., Thomas, J., Brooker, D., \& Casey, D. (2017). From screening to synthesis: Using nvivo to enhance transparency in qualitative evidence synthesis. Journal of Clinical Nursing, 26(5/6), 873-881. https://doi.org/101111/jocn.13443

Hurt, C.S., Cleanhous, S., \& Newman, S.P. (2017). Further explorations of illness uncertainty: Carers' experiences of Parkinson's disease. Psychology \& Health, 32(5), 549-566. https://doi.org/10.1080/08870446.2017.1283041

Inan, F. S., Gunusen, N. P., \& Ustun, B. (2016). Experiences of newly diagnosed breast cancer patients in Turkey. Journal of Transcultural Nursing, 27(3), 262-269. https://doi.org/10.1177/1043659614550488

Jeon, B. H., Choi, M., Lee, J., \& Noh, S. H. (2016). Relationship between gastrointestinal symptoms, uncertainty, and perceived recovery in patients with gastric cancer after gastrectomy. Nursing \& Health Sciences, 18(1), 23-29. https://doi.org/10.1111/nhs.12219

Jiang, X., \& He, G. (2012). Effects of an uncertainty management intervention on uncertainty, anxiety, depression, and quality of life of chronic obstructive pulmonary disease outpatients. Research in Nursing \& Health, 35(4), 409-418. https://doi.org/10.1002/nur.21483

Kim, J., McMahon, B., Hawley, C., Brickham, D., Gonzalez, R., \& Lee, D. (2016). Psychosocial adaption to chronic illness and disability: A virtue-based model. Journal of Occupational Rehabilitation, 26(1), 45-55. https://doi.org/10.1007/s0926-015-9622-1

Kornblith, A. B., Powell, M., Regan, M. M., Bennett, S., Krasner, C., Moy, B., Younger, J., Goodman, A., Berkowitz, R., \& Winer, E. (2007). Long-term psychosocial adjustment ofolder versus younger survivors of breast and endometrial cancer. Psycho-Oncology, 16, 895-903. https://doi.org/10.1002/pon.1146 
Kurita, K., Garon, E. B., Stanton, A. L., \& Meyerowitz, B. E., (2013). Uncertainty and psychological adjustment in patients with lung cancer, Psycho-Oncology, 22, 1396-1401. https://doi.org/10.1002/pon.3155

Martinez-Marcos, M., \& De la Cuesta-Benjamea, C. (2015). Women's self-management of chronic illnesses in the context of caregiving: A grounded theory study. Journal of Clinical Nursing, 24(11/12), 15571566. https://doi.org/10.1111/jocn.12746

Mishel, M. H. (1981). The measurement of uncertainty in illness. Nursing Research, 30(5), 258-263.

Mishel, M. H. (2014). Theories of uncertainty in illness. In M. J. Smith \& P. R. Liehr (Eds), Middle range theory for nursing (3rd ed., pp. 53-86). Springer.

Nanton, V., Munday, D., Dale, J., Mason, B., Kendall, M., \& Murray, S. (2016). The threatened self: Considerations of time, place, and uncertainty in advanced illness. British Journal of Health Psychology, 21(2), 351-373. https://doi.org/10.1111/bjhp.12172

Polit, D. F., \& Beck, C. T. (2012). Nursing research: Generating and assessing evidence for nursing practice (9th ed.). Lippincott Williams \& Wilkins.

Sajjadi, M., Rassouli, M., Bahri, N., \& Mohammadipoor, F. (2015). The correlation between perceived social support and illness uncertainty in people with human immunodeficiency virus/acquired immune deficiency syndrome in Iran. Indian Journal of Palliative care, 21(2), 231-235. https://doi.org/10.4103/0973-1075.156508

Sav, A., King, M. A., Whitty, J. A., Kendall, E., McMillan, S. S., Kelly, F., Hunter, B., \& Wheeler, A. J. (2015). Burden of treatment for chronic illness: A concept analysis and review of literature. Health Expectations, 18(3), 312-324. https://doi.org/10.1111/hex.12046

Suzuki, M. (2012). Quality of life, uncertainty, and perceived involvement in decision making in patients with head and neck cancer. Oncology Nursing Forum, 39(6), 541-548. https://doi.org/10.1188/12.0NF.541-548

\section{[Appendix follows]}




\section{Appendix}

\section{Nodes and Main Themes}

Node 1: Chronic Illness

- Diagnosis or diagnoses

- Length of time to diagnosis

- Length of time diagnosed

Node 2: Uncertainty

- Experiences with uncertainty

- Uncertainty related to

- searching for diagnosis

- procedure for diagnosis

- treatment

- outcomes

○ the future

Node 3: Impact of Uncertainty on Daily Life

- Psychological impact

- Planning daily activities

- Seclusion/fear of leaving the house

- Family impact

- Inability to work

Node 4: Coping or Managing Uncertainty

- Finding routine

- Physical activity
- Spiritual rituals

- Researching

- Inability to mange

- Therapy

- Plan ahead

- Incorporate resting periods

Node 5: Communication With Healthcare Team About Uncertainty

- Trust in doctors

- Lack of information provided by healthcare team

- Unclear information provided by healthcare team

- Guidance from healthcare team

- Therapist referrals to assist in healthcare team communication

\section{Node 6: Important Information From}

Participants

- Have an advocate

- Treating each case differently

- Improve explanations of treatments plans

- Getting to know patients 